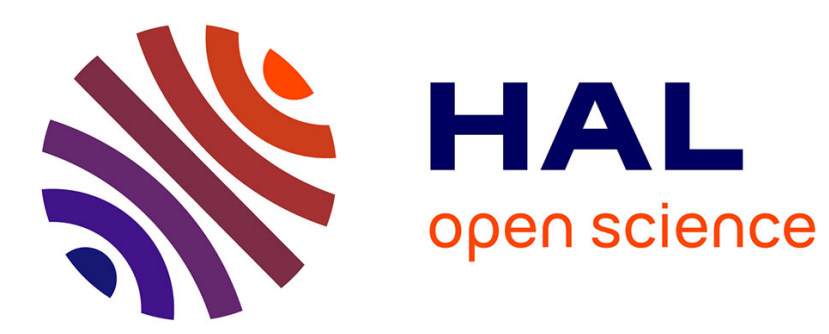

\title{
Aperçu sur les débouchés actuels du bois de douglas
}

Roger Lebrun

\section{To cite this version:}

Roger Lebrun. Aperçu sur les débouchés actuels du bois de douglas. Revue forestière française, 1963, 10, pp.785-790. 10.4267/2042/24500 . hal-03386158

\section{HAL Id: hal-03386158 https://hal.science/hal-03386158}

Submitted on 19 Oct 2021

HAL is a multi-disciplinary open access archive for the deposit and dissemination of scientific research documents, whether they are published or not. The documents may come from teaching and research institutions in France or abroad, or from public or private research centers.
L'archive ouverte pluridisciplinaire HAL, est destinée au dépôt et à la diffusion de documents scientifiques de niveau recherche, publiés ou non, émanant des établissements d'enseignement et de recherche français ou étrangers, des laboratoires publics ou privés. 


\title{
APERÇU SUR LES DÉBOUCHES ACTUELS DU BOIS DE DOUGLAS
}

\author{
PAR \\ R. LEBRUN \\ Ingénieur Principal des Eaux et Forêts \\ à Guebwiller
}

Les premiers Pseudotsuga Douglasii ont été introduits en Alsace depuis bientôt 80 ans. Les plus vieux peuplements ont été constitués vers 1885 aux environs de Barr et en 1891 dans la forêt domaniale de Guebwiller, à proximité de la maison forestière de la Rolle. Dans ce dernier cas il est difficile de parler de peuplement puisqu'il est réduit à 17 individus qui ont été plantés en mélange avec des épicéas sur une surface de quelques ares, mais ces sujets sont tellement beaux qu'ils méritent d'être distingués. Les archives ont conservé le nom de l'ouvrier forestier KoHLER qui a procédé à la plantation. Les conditions favorables:

$$
\begin{array}{ll}
\text { altitude } & : 850 \mathrm{~m} . \\
\text { sous-sol } & : \text { grauwacke } \\
\text { sol } & : \text { très profond (ancien pâturage) } \\
\text { pluvionétrie } & : 1200 \mathrm{~mm} \\
\text { exposition } & : \text { Est, abritée }
\end{array}
$$

ont permis une croissance remarquable.

Avec une hauteur de 43 à $50 \mathrm{~m}$ et une végétation encore très active qui leur permet de faire une pousse annuelle terminale de quelques dizaines de centimètres, ces douglas doivent dans quelques années détenir le record du continent. Ils ne seraient surclassés en Europe que par leurs collègues écossais.

Parmi ces quelques sujets, notre camarade Bouvarel, il y a quelques années, a sélectionné $G 1$ et $G 2$ comme étalons de vergers à graines. Après une nouvelle reconnaissance effectuée par 
M. 1'Ingénieur LACAZE un nouvel arbre + G 3 a été retenu, cet arbre est vraiment remarquable par le port original que lui donnent ses rameaux exceptionnellement courts. Cette particularité ainsi que les caractères de noblesse de sa race donneront à cette souche un intérêt certain pour des stations qu'il restera à préciser, sa vocation sera peut-être les reboisements d'altitude.

Depuis l'époque de ces premiers essais les plantations se sont poursuivies dans la région. De 1900 à 1914, des peuplements ont été ainsi créés sur des surfaces de quelques hectares au total clans plusieurs forêts communales : Soultz, Soultzmatt, Wasserbourg, Rouffach, Pfaffenheim. Par bonheur il s'agissait de graines de très bonnes races qui avaient été distribuées dans les différentes pépinières. La règle à cette époque était en effet que chaque triage dispose pour chaque forêt d'une petite pépinière. Ces peuplements, après quelques éclaircies assez fortes, sont tous remarquables, une première prospection a permis de retenir 7 placeaux pour la récolte de graines, 5 d'entre eux sont particulièrement beaux. Plusieurs dizaines d'arbres + peuvent être facilement choisis.

Les plantations n'ont pas été interrompues par la première guerre mondiale, puisqu'au contraire c'est grâce à des plants récupérés dans cles pépinières situées dans les territoires occupés en Belgique par l'Armée allemande que de très beaux peuplements ont été constitt:és en 1917 dans les forêts communales de Guebwiller, Pfaffenheim et en forêt domaniale.

Ces plants étaient également de très bonne race, toutefois leur croissance a été un peu moins active. Est-ce du fait de l'origine des graines ou parce qu'ils ont été utilisés pour créer des peuplements purs à forte densité $1,20 \mathrm{~m} \times 1,20 \mathrm{~m}$, alors que précérlemment les clouglas avaient été plutôt introduits en mélange avec le sapin et l'épicéa ou bien encore parce que les éclaircies ont été trop tarclives ou trop prudentes?

Il est difficile pour le moment de déterminer l'influence de ces différents facteurs. Grâce à des éclaircies répétées, il sera plus commorle dans une dizaine d'années d'arrêter une opinion.

Après 1920, l'introduction s'est poursuivie jusqu'en 1940, mais i! semble que les graines étaient d'origine plus variées, à côté de plants le très bonne venue, il apparait que quelques lots sont douteux arec des verticilles très obliques. Toutefois. on peut avoir dès à présent l'assurance que beaucoup de peuplements créés pendant cette périole auront une classe identique à celle de leurs ainés.

Pendant la seconcle guerre mondiale les graines ont fait défaut. mais dè: 1950 , grâce à cles récoltes effectuées sur les meilleurs porto-graines cle la région, cette essence a vu augmenter son importance ilans les reboisements et depuis le douglas tient la vedette.

Cette préférence se justifie parce que malgré sa rapidité de croissance, il conserve dans les. Vosges Alsaciennes, dans des stations 


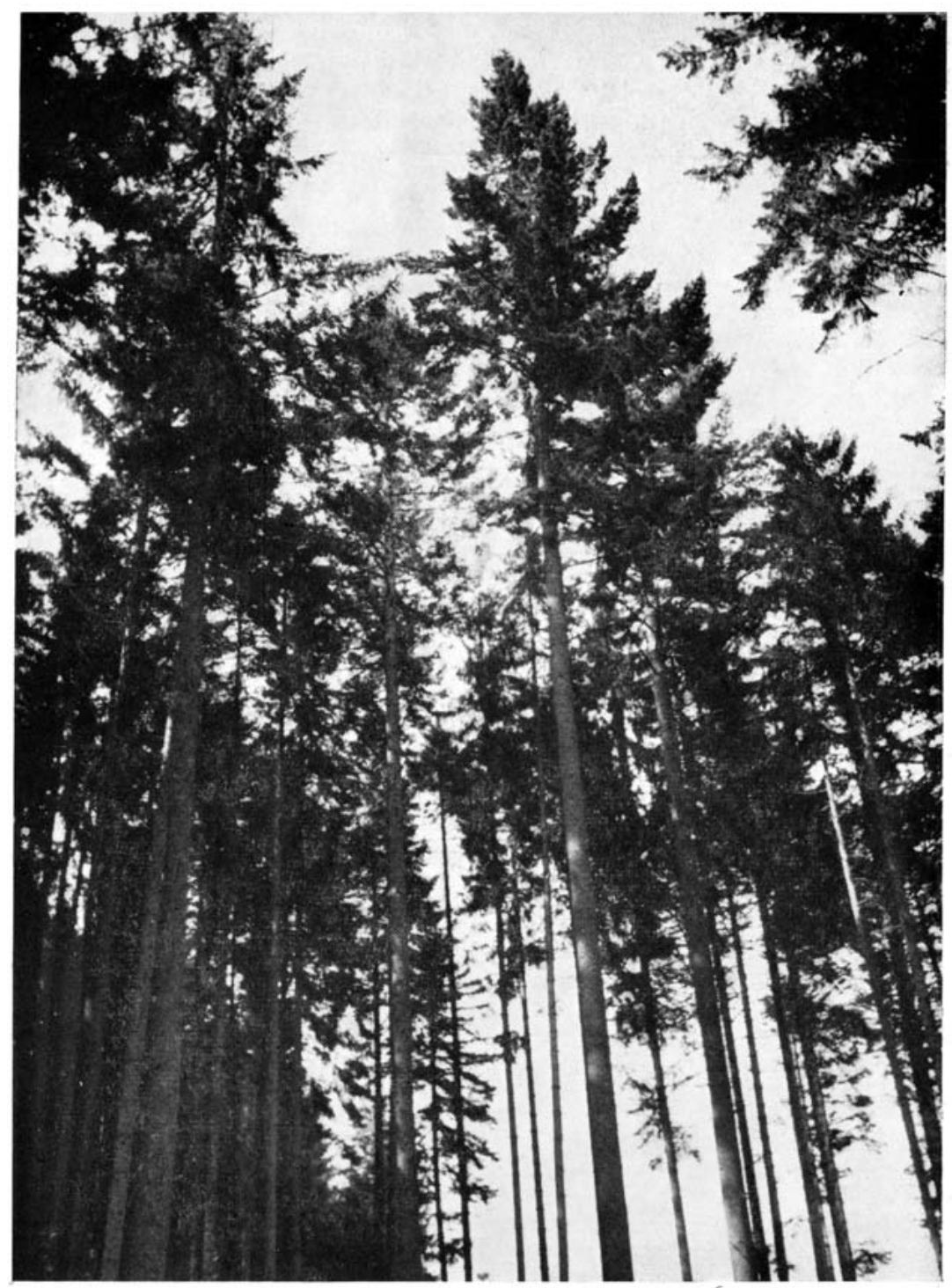

Voici l'arbre « plus 》 GBW3, choisi en particulier pour la finesse de ses branches et l'élégance de son port.

(Cliché J. Kirchmeyer.) 


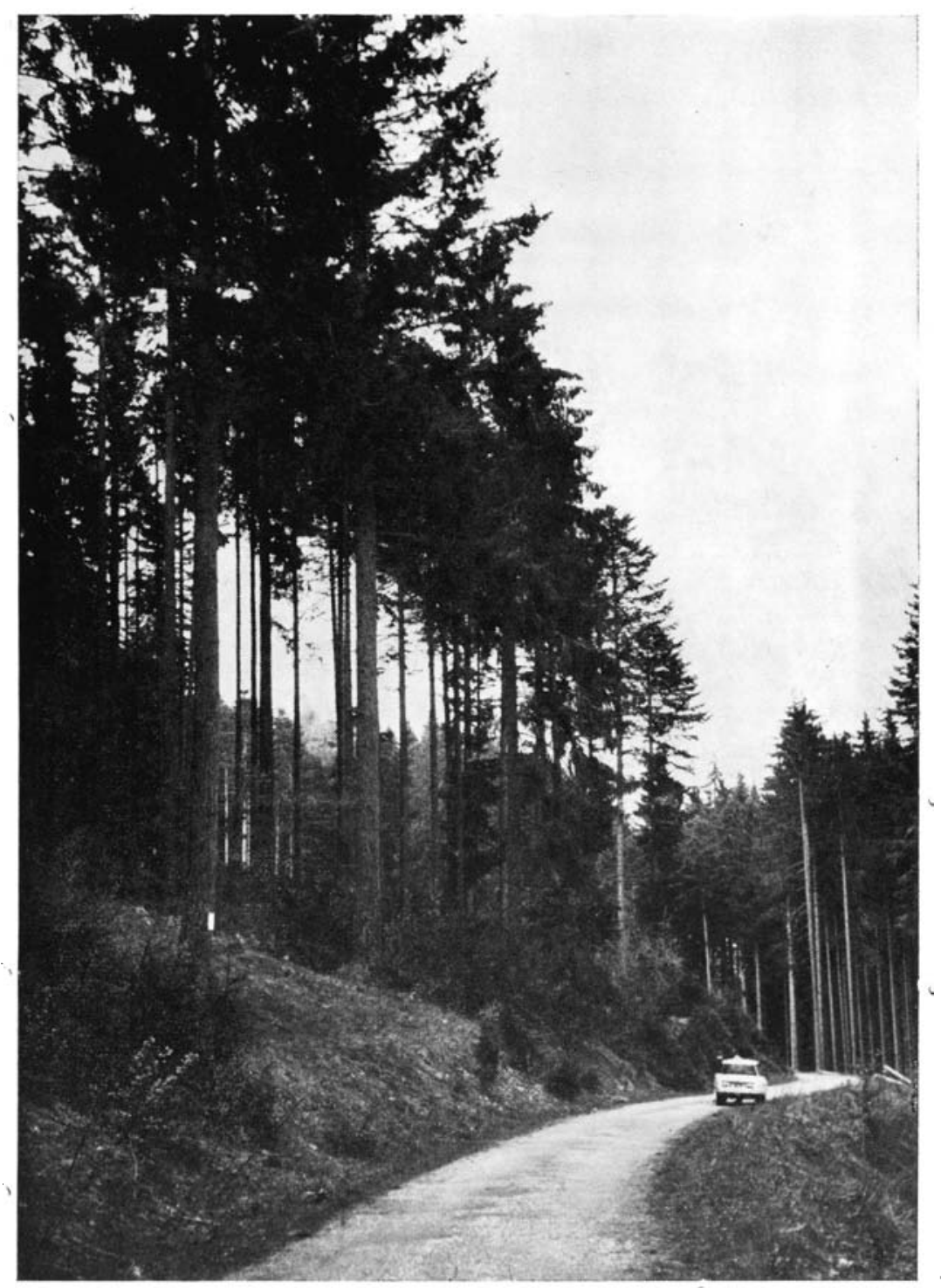

Au-dessus de la Route forestière de la Rolle quelques-uns des 17 douglas plantés en 1891.

(Cliché J. Kirchmeyer.) 
très variées à des altitudes comprises entre 300 et $850 \mathrm{~m}$, quelle que soit l'exposition, des qualités constantes qui le distinguent des peuplements d'autres régions. Les branches sont fines, l'élagage est fort convenable et en tout cas nettement meilleur que celui des sapins ou des épicéas voisins, au même âge, ses accroissements sont réguliers. D'autre part, jusqu'à présent du moins, il ne connaît pas d'ennemis aussi redoutables que ceux qui détruisent périodiquement les sapins et les épicéas. Actuellement, dans l'Inspection de Guebwiller, les surfaces des peuplements constitués pour l'essentiel par le douglas se répartissent comme suit:

$$
\begin{aligned}
& \text { plus de } 50 \text { ans : } 15 \text { ha } \\
& 31 \text { à } 50 \text { ans }: 27 \text { ha } \\
& 11 \text { à } 30 \text { ans }: 23 \text { ha } \\
& 1 \text { à } 10 \text { ans : } 160 \text { ha }
\end{aligned}
$$

Si la cadence actuelle se maintient, la production interviendra sur le marché avec une importance croissante, c'est pourquoi il apparaît utile de s'interroger sur les résultats qu'il est permis d'escompter et en particulier de se soucier de l'intérêt et de la valeur des produits qui dans l'état actuel des techniques intéresseront successivement :

- les usines de râperie,

- les utilisateurs de petites grumes (bois de soutènement, poteaux de lignes),

- les scieries.

\section{La râperie}

C'est dans cette industrie que les produits trouveront le plus rapidement un débouché normal. C'est aussi malheureusement dans ce secteur que le douglas est le moins favorisé. En effet, dans le courant de ces dernières années quelques centaines de stères ont été fournis à la papeterie et en particulier à la "Cellulose de Strasbourg " qui a entrepris des essais industriels et qui poursuit ces essais en laboratoire. Cette société a bien voulu, à l'intention de cet article, faire le point des résultats qu'elle a obtenus.

Le procédé classique au bisulfite de calcium convient mal au douglas pour les raisons suivantes:

- la consommation de réactif de cuisson (chaux) est anormalement forte,

- - la proportion des incuits est particulièrement élevée, ce qui a pour corollaire une diminution des rendements en bonne pâte et un ralentissement de la chaine de fabrication, 
- la pâte à l'état écru a une coloration prononcée en jaune paille, ce qui exclut pratiquement son emploi en papeterie autrement qu'à l'état blanchi.

Il est alors nécessaire d'envisager d'autres techniques de cuisson. Une solution semble pouvoir être trouvée en remplaçant, dans la lessive, le calcium par une base soluble (sodium, magnésium, ammonium). Mais si ce procédé marque un progrès considérable pour les pins, dans les premiers essais confirmés à l'échelle industrielle dans une entreprise de l'Ontario, les résultats obtenus avec le douglas sont moins favorables.

En conclusion, il est probable que l'utilisation industrielle du douglas pour la papeterie exigera la mise au point d'une technique qui lui sera propre, ce qui entraînera des dépenses d'investissement qui ne seront rentables que lorsque les quantités offertes sur le marché seront importantes.

Pendant toute cette période transitoire, les seuls débouchés pour le bois de douglas seront les pâtes Kraft dont il constitue la matière première de grande qualité ou les panneaux de particules, c'est-à-dire que les prix obtenus seront dans le même rapport que celui qui apparaît dans le tableau annexé, à savoir entre le tiers et les deux tiers de la valeur du stère de sapin ou d'épicéa.

Cette différence de prix est l'une des raisons pour laquelle il est préférable de planter le douglas en mélange avec des épicéas qui seuls sont intéressés par les premières éclaircies, plutôt que de constituer des peuplements purs qui de toute façon seraient d'une création plus onéreuse et d'une éducation plus délicate.

\section{Les utilisateurs de petites grumes}

Quel est l'intérêt du douglas comme bois de soutènement et comme poteau de ligne? M. l'Ingénieur en Chef Venet, au cours des travaux qu'il a conduits pour son " Etude de la résistance mécanique des bois de mine en fonction des facteurs de la production forestière ") (1), a communiqué le résultat des essais qu'il a effectués sur 3 lots de sapin, d'épicéa et de douglas qui provenaient de la même station, à savoir la parcelle 143 de la forêt domaniale de Guebwiller. Cette étude a mis en évidence que lorsque le douglas a poussé en montagne ses qualités sont tout à fait comparables à celles des résineux indigènes, puisque sa résistance le classe après le sapin mais avant l'épicéa.

M. VENET poursuit actuellement des essais sur l'utilisation du douglas pour la production de poteaux de ligne. Les bois proviennent d'un peuplement de 45 ans (parcelle 8 de la forêt domaniale

(1) Revue Forestière, $\mathrm{n}^{\bullet} 12$, décembre 1958. 
de Guebwiller). Il ne fait pas de doute que les résultats confirmeront ceux qui ont été obtenus pour les bois de soutènement. L'ostracisme qui frappait le douglas pour cet emploi est de la même veine que les mesures contenues dans certains cahiers des charges qui excluaient de la construction les bois français, quelles que soient leur origine et leur qualité.

Il y a lieu de noter que dans la plupart des peuplements de la région la décroissance du douglas est comparable à celle du sapin. C'est pourquoi, malgré le risque de difficultés que les utilisateurs pouvaient soulever s'ils reconnaissaient le douglas, ce qui ne doit pas être fréquent, les acheteurs ont fait pour les lots de poteaux de douglas des offres voisines des cours des poteaux de sapin ou d'épicéa.

Pour ce débouché, il ne semble donc pas que la vente du douglas soulève des difficultés particulières. Il s'agit malheureusement d'un marché qui risque de connaitre la stagnation dans un proche avenir.

\section{Les scieries}

Jusqu'à présent, les scieurs ont offert exactement le prix qui correspondait à la catégorie des grumes mises en vente sans faire de distinction entre le douglas et le sapin ou même l'épicéa. En outre, certains lots ont été spécialement recherchés par des menuisiers qui apprécient ce bois car ils ont reconnu le Pin d'Orégon, nom sous lequel le Douglas d'Amérique est commercialisé. Les grumes qu'ils achètent sont évidemment celles d'un diamètre au moins égal à $0,40 \mathrm{~m}$, c'est-à-dire celles qui leur fournissent une proportion notable de bois de qualité entre l'aubier et la partie du cœur où les accroissements sont trop forts et où les nœuds existent. Les quantités offertes sont très modestes, mais il semble que dans l'avenir le douglas de la qualité produite dans cette région aura plutôt un léger avantage sur le sapin, ou pour le moins une valeur égale. Il ne faudrait pas en conclure que cette parité sera réalisée quelle que soit la station dans laquelle aura crû le douglas et quelle que soit sa race. Il est évident qu'une décote considérable frappera les peuplements mal élagués ou d'accroissement trop exubérant.

Ces quelques renseignements permettent de penser que le douglas aura en économie forestère un rôle comparable à celui que l'on a coutume d'obtenir avec les produits ou les méthodes qui comme lui ont franchi l'Atlantique. La ressource qu'il apportera, sauf dans les stations privilégiées, qui sont nombreuses dans les Vosges alsaciennes, ne sera pas d'une qualité supérieure à celle des essences du vieux continent, mais elle aura l'avantage d'être disponible plus rapidement, ce qui augmentera le profit des propriétaires en même temps que l'industrie du bois disposera d'une matière première plus abondante. 


\section{Prix obtenus pour des lots de douglas homogènés vendus après façonnage}

\begin{tabular}{|c|c|c|c|c|}
\hline Date & Volume & Nombre & Prix du & Prix du sapin \\
\hline & $\mathrm{m}^{3} \quad \mathrm{st}$ & de pièces & & aux mêmes ventes \\
\hline - & - & - & - & - \\
\hline 7.07 .1955 & 25 st. & - & 1730 anc. $F$ & 3238 anc. $F$ \\
\hline 27.10 .1955 & 27 st. & - & 2051 anc. $F$ & 3285 anc. $F$ \\
\hline 27.10 .1955 & 48 st. & - & 2024 anc. $F$ & 3285 anc. $F$ \\
\hline 20.12 .1955 & 55 st. & - & 2034 anc. $\mathrm{F}$ & 3256 anc. $F$ \\
\hline 25.09 .1962 & 20 st. & - & $16,96 \mathrm{~F}$ & $45,05 \mathrm{~F}$ \\
\hline 25.04 .1956 & $14,97 \mathrm{~m}^{3}$ & 130 & 3548 anc. $\mathrm{F}$ & 4028 anc. F \\
\hline 27.11.1957 & $10,46 \mathrm{~m}^{3}$ & 10 & 5942 anc. $F$ & 5742 anc. $F$ \\
\hline 29.06 .1961 & $40,90 \mathrm{~m}^{3}$ & 45 & $95,37 \mathrm{~F}$ & $96,14 \mathrm{~F}$ \\
\hline 22.03 .1962 & $47,21 \mathrm{~m}^{3}$ & 222 & $70,73 \mathrm{~F}$ & $90,03 \mathrm{~F}$ \\
\hline 29.05 .1962 & $66,83 \mathrm{~m}^{3}$ & 222 & $81,84 \mathrm{~F}$ & $96,90 \mathrm{~F}$ \\
\hline 29.05 .1962 & $57,67 \mathrm{~m}^{3}$ & 81 & $89,33 \mathrm{~F}$ & $96,90 \mathrm{~F}$ \\
\hline 29.05 .1962 & $28,38 \mathrm{~m}^{3}$ & 26 & $95,99 \mathrm{~F}$ & $96,90 \mathrm{~F}$ \\
\hline
\end{tabular}

